\title{
INTERFEROMETRIC SEA ICE MAPPING WITH TANDEM-X: FIRST EXPERIMENTS
}

\author{
R. Scheiber, F. De Zan, P. Prats, L. Sant'Anna Araújo, M. Künemund, L. Marotti \\ Microwaves and Radar Institute, German Aerospace Center (DLR) \\ P.O. Box 1116, 82230 Wessling, Germany \\ Tel.: +49 - 8153282319, E-mail: rolf.scheiber@dlr.de
}

\begin{abstract}
In summer 2010 during the commissioning phase of TanDEM-X, the TerraSAR-X/TanDEM-X constellation was operated in the pursuit monostatic mode allowing alongtrack interferometric measurements with very short time separation of only $2.6 \mathrm{sec}$. This paper presents first evaluations of data acquired on arctic sea ice scenarios and investigates the potentials for the mapping of ice sheet dynamics with respect to rotation and translation with this unique constellation. Due to the larger coverage, the data analysis is focused on interferometric ScanSAR data.
\end{abstract}

Index Terms - SAR interferometry, sea ice, ocean currents, rotation, translation

\section{INTRODUCTION}

The monitoring of sea ice coverage and type from space is a routine service implemented with the support of several space agencies (e.g. ESA, CSA, DLR). Due to its weather independence synthetic aperture radars like ESA's ASAR on-board ENVISAT and CSA's Radarsat-2 are sensors of first choice allowing wide swath coverage of several hundreds of $\mathrm{km}$ at resolutions in the order of $10-50 \mathrm{~m}$ in ScanSAR mode. With the German TanDEM-X launched in June 2010, the very first short-time interval interferometric SAR mapping opportunities became realistic. The TanDEM-X mission was designed with the primary goal to generate a consistent high-resolution, world-wide digital elevation model [1]. For this purpose two nearly identical spaceborne SAR sensors are operated in close formation in bistatic mode of operation, exploiting the across-track baselines as given by the Helix formation. Secondary mission objectives include the monitoring of different moving objects by means of along-track interferometry. Depending on the along-track baseline, different sensitivities can be selected according to applications like moving target detection/traffic monitoring, sea currents or ice drift measurements. Due to water surface decorrelation, short baselines/time intervals in the order of few tens of meters/milliseconds are required for ocean current measurements. However, sea ice drift can be measured with much larger baselines in the order of several tens of $\mathrm{km}$ (corresponding to seconds). During the commissioning phase of the TanDEM-X satellite the twin satellites were operated in the so-called pursuit monostatic mode with an along-track baseline of $20 \mathrm{~km}$, corresponding to 2.6 seconds time interval, which was expected to provide sufficient sensitivity to ice drift measurements. Due to its increased swath coverage, the ScanSAR mode has been selected to acquire first dedicated data sets in arctic regions.

In this paper it is shown that through interferometric analysis stable ice sheets can be distinguished from floating ice bergs and highly accurate quantitative measurements of ice sheet rotation and translation are possible.

\section{MEASUREMENTS OF SEA ICE DYNAMICS}

The direction and velocity of ocean currents can be inferred from the motion of the sea ice cover, in particular in the transition zone of open water and closed sea ice cover. In this zone ice sheets of different size and number are present. In general the motion of each ice sheet can be characterized by translation and rotation around different axis, among which the rotation around its vertical axis is the most likely and prominent one, as already shown in [2] and to be confirmed in the next subsections.

\subsection{Ice Drift}

Ice drift can generally be inferred from co-registration measurements of two data takes (not necessarily interferometric ones) acquired at different time instants. For TanDEM-X the across-track displacements could be refined from the interferometric phase making use of its much higher accuracy. However, first measurements with TanDEM-X indicated the presence of ice sheet rotation [3], which complicates the interferometric analysis, unless it can be properly considered. Therefore, ice drift measurements are performed via coregistration estimates by means of spectral diversity, a technique allowing precise and spatially adaptive estimates [4]. 


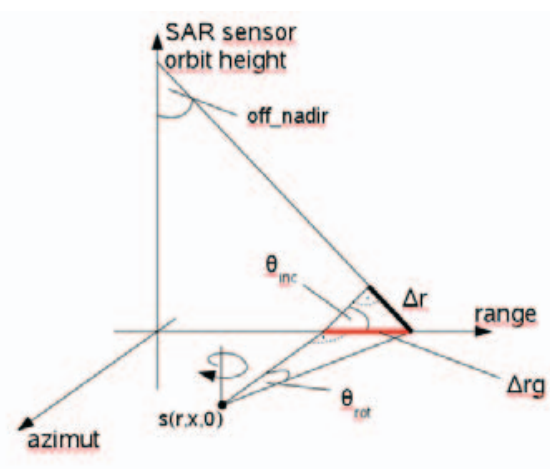

Fig.1: Ice sheet rotation around vertical axis.

It is applied in range and azimuth directions, whereas in azimuth, proper consideration of the ScanSAR signal characteristics is required. Considering that the ice drift within few seconds will be in the order few meters at maximum, sub-pixel accuracies in the order of $1 / 100$ of a resolution cell are required, especially for the ScanSAR case with only $12 \mathrm{~m}$ azimuth resolution. To achieve this accuracy, strong spatially adaptive averaging is required by considering the influence of ice sheet boundaries and their rotation.

\subsection{Ice Sheet Rotation}

The presence of sea ice sheets characterized by along-track fringes in SAR interferograms has first been reported by M. Thomas et al [2]. They investigated ERS-1/ERS-2 data acquired at 1 day time interval. The authors explained the individual fringe patterns as a differential interferometric effect, induced by the rotation of the ice blocks about their vertical axis. The interferometric phase as a function of azimuth position $x$ from the center of mass (rotation) of each ice sheet is:

$$
\phi_{i f}(x)=4 \pi / \lambda \cdot x \cdot \sin \theta_{r o t} \cdot \sin \theta_{i n c},
$$

where $\theta_{\text {inc }}$ is the local incidence angle and $\theta_{\text {rot }}$ is the ice sheet rotation angle (see Fig.1). Our analysis of TanDEM-X interferograms confirms this hypothesis.

We propose to perform the numerical and spatially adaptive evaluation of the fringe rate by investigating the phase of the autocorrelation function for lag one of the complex interferogram, $R_{l}$. This has previously been proposed for the estimation of the Doppler centroid in [4] or for the estimation of the interferometric phase slopes. The estimation of the rotation angle is given by

with

$$
\theta_{r o t}(r, x)=\frac{\lambda \cdot \operatorname{atan} R_{1}(x)}{4 \pi \cdot \Delta x \cdot \sin \theta_{i n c}(r)}
$$

$$
R_{1}(x)=\sum_{-x_{0}-n \Delta x}^{-x_{0}-n \Delta x} \exp \left(j \phi_{i f}(x)\right) \cdot \exp \left(-j \phi_{i f}(x-\Delta x)\right),
$$
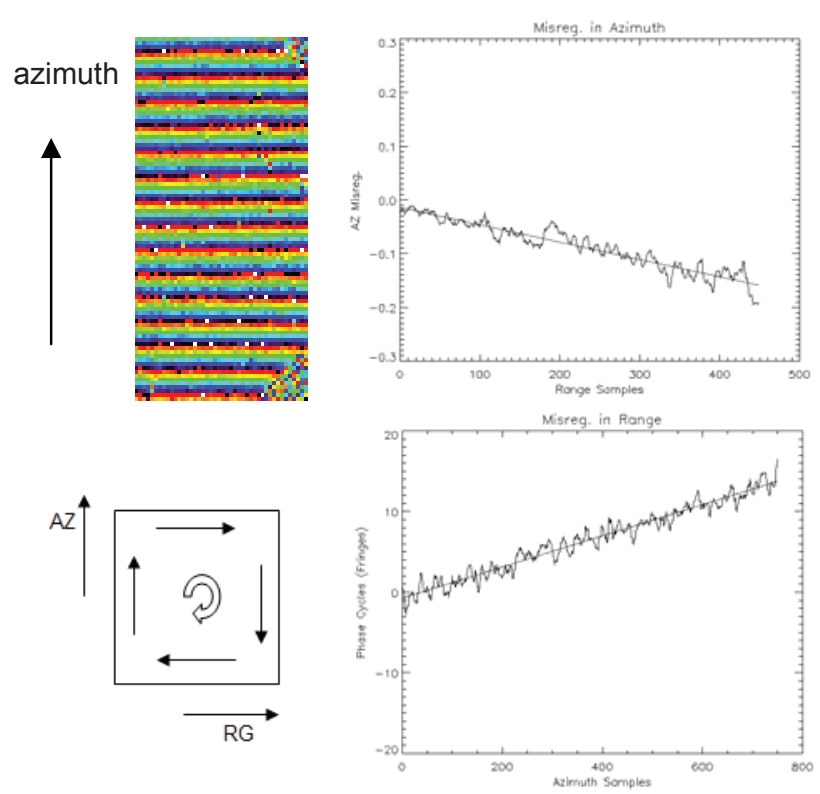

Fig.2: Validation of the rotation hypothesis: (left) azimuth fringe pattern of a selected ice sheet and anticipated rotation. (right) variable coregistration estimates for azimuth (top) and range (bottom) supporting the rotation hypothesis. Range misregistration is transformed to interferometric phase cycles for validation purposes.

The measured fringe rates translate into rotation angles in the order of few thousands of a degree in the 2.6 seconds between the two acquisitions. The maximum rotation angles are determined by decorrelation effects due to the limited azimuth bandwidth/resolution. Total decorrelation occurs for rotation angles of $\lambda /\left(2 \rho_{x} \sin \theta_{i n c}\right)$, where $\rho_{x}$ is the azimuth resolution. For TanDEM-X ScanSAR data this corresponds to $+/-0.086 \mathrm{deg}$ and for stripmap data to $+/-0.25$ deg at 30 deg incidence angle.

\subsubsection{Validation of Rotation Hypothesis}

Coregistration estimates were performed for an ice sheet of a stripmap data set in order to validate the rotation hypothesis (stripmap data are preferred for this investigation because of the better azimuth resolution).

Results are shown in Fig. 2 indicating a clock-wise rotation around the vertical axis. The measured range misregistration matches the number of fringes of the selected block.

\subsubsection{Discussion}

At this point it is important to remark that in principle the rotation of the ice sheets also occurs around the horizontal axis perpendicular to the ground track, which might lead to a similar interferometric azimuth phase pattern, or around the horizontal axis parallel to the ground track, which would generate a phase pattern rotated by 90 degrees. Both types of rotation lead to spectral decorrelation, similar to the well known baseline decorrelation phenomenon. Assume a 
rotation about an axis parallel to the ground track of $0.1 \mathrm{deg}$. This would correspond to an equivalent baseline of $1 \mathrm{~km}$ at the orbit height of $540 \mathrm{~km}$, which is one fifth of the critical baseline of TerraSAR-X. Indeed, decorrelation effects can be observed especially for isolated ice sheets in the open sea, which are attributed to horizontal rotation above the correlation limits.

\subsection{Ice Sheet Segmentation}

Based on the generated rotation map, a segmentation of individual ice sheets is possible. First the completely decorrelated open water areas are masked by coherence thresholding. If possible, also the stable land areas should be excluded from the evaluation, as their topographic interferometric phase component leads to invalid results. Based on the erosion and the dilatation morphological operators, ice sheet regions above a certain minimum size are marked as valid and subsequently individual ice sheets are identified by region growing using a blob coloring algorithm [6]. The output is a list of valid (sufficiently large), isolated ice sheets, characterized by high coherence and closed contours. For each ice sheet the center of mass is computed to serve as the center of rotation.

Finally, each ice sheet is annotated by its mean rotation angle and the $2 \mathrm{D}$ range/azimuth translation vector. Special care is taken for the computation of the mean range/azimuth displacement between the two acquisitions. Instead of simple averaging or just taking the noisy displacement in the center of mass we decided to correct for the linear trends caused by the ice sheet rotation prior to averaging (see Fig. 3 ). This is especially important for coregistration estimates obtained from the phase by means of spectral diversity and avoids bias caused by wrap-around effects.

\section{NORTH-EAST GREENLAND TEST CASE}

One of the first TerraSAR-X/TanDEM-X interferometric ScanSAR scenes has been acquired at the NE coast of Greenland on August 2, 2010. The data were processed with the experimental TanDEM-X Interferometric Processor (TAXI) [7], which uses the baseband azimuth scaling (BAS) algorithm for the processing of the ScanSAR data [8]. Fig. 4 presents the results of the second subswath of this ScanSAR data take. The imaged area includes some islands (stable, but with topography), ice shelves (stable and mostly flat) and floating ice sheets (drifting and rotating). The rotation induced azimuth fringe rates are clearly visible in the interferometric phase image, allowing directly the identification of unstable ice regions. The rotation map quantifies the rotation angles around the vertical axis to values between $-0.005 \mathrm{deg}$ to $+0.005 \mathrm{deg}$. After segmentation the smaller ice sheets are discarded (see Fig. 4(d)). For the larger ice sheets the estimation of the mean rotation and translation is possible. The $2 \mathrm{D}$ translation map is depicted in

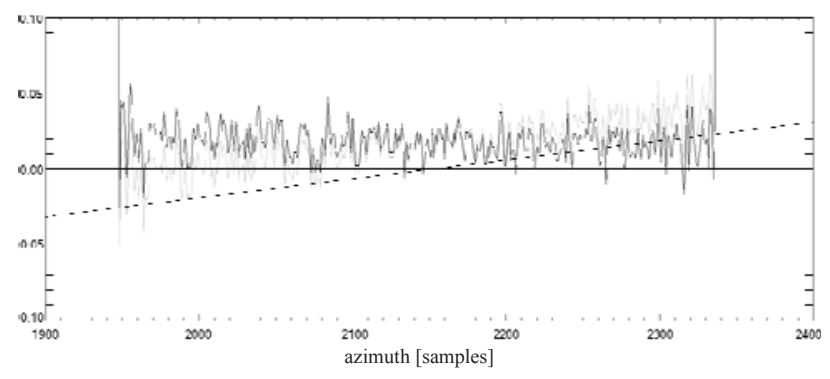

Fig.3: Range displacement in samples along azimuth for a specific ice sheet. The center of mass is located at azimuth sample \#2150. The mean rotation (straight solid line) is used to correct the initial range registration estimates (noisy light gray line) to obtain unbiased estimates (noisy solid line) prior to averaging. The mean displacement for this ice sheet is 0.02 slant range samples, corresponding to $3.6 \mathrm{~cm}$.

Fig.4(e). The arrows indicate the direction and magnitude of the ice drift, whereas the colors quantify only the magnitude between $0 \mathrm{~m}$ and $0.5 \mathrm{~m}$.

\section{CONCLUSIONS}

The results achieved so far will be used to re-adjust and possibly increase the along-track baseline to allow ice drift measurements with better accuracy during the next pursuit monostatic period, which is scheduled at the end of the nominal TanDEM-X mission life-time.

The first interferometric measurements of sea ice dynamics obtained by TanDEM-X show a first glimpse of the potential of interferometric radar constellations for monitoring highly dynamic processes in the Earth's cryosphere. For the first time extensive observations of sea ice sheet rotation and translation became possible. Regular acquisitions are expected to help for a better understanding of the dynamics of sea ice processes in the Arctic regions.

\section{REFERENCES}

[1] G. Krieger., A. Moreira, H. Fiedler, I. Hajnsek, M. Werner, M. Younis, and M. Zink: "TanDEM-X: A Satellite Formation for High-Resolution SAR Interferometry“, IEEE Trans. Geosci. Remote Sens., vol. 45, no. 11, pp. 3317-3341, November, 2007.

[2] M. Thomas, M. Steffens, and R. Roth: "Measuring changes of iceberg attitudes by SAR interferometry", Proc. of 3rd ERS symposium, Florence, 1997 (http://earth.esa.int/workshops/ers97/papers/thomas1/)

[3] F. Lopez-Dekker, P. Prats, F. De Zan, Daniel Schulze, Gerhard Krieger, Alberto Moreira, ,TanDEM-X first DEM acquisition: a crossing orbit experiment," to be published in Geosci. and Remote Sens. Letters, 2011.

[4] R. Scheiber., A. Moreira: "Coregistration of Interferometric SAR Images using Spectral Diversity “, IEEE Trans. Geosci. Remote Sens., vol. 38 pp., no. 5, pp. 2179-2191, September, 2000. 


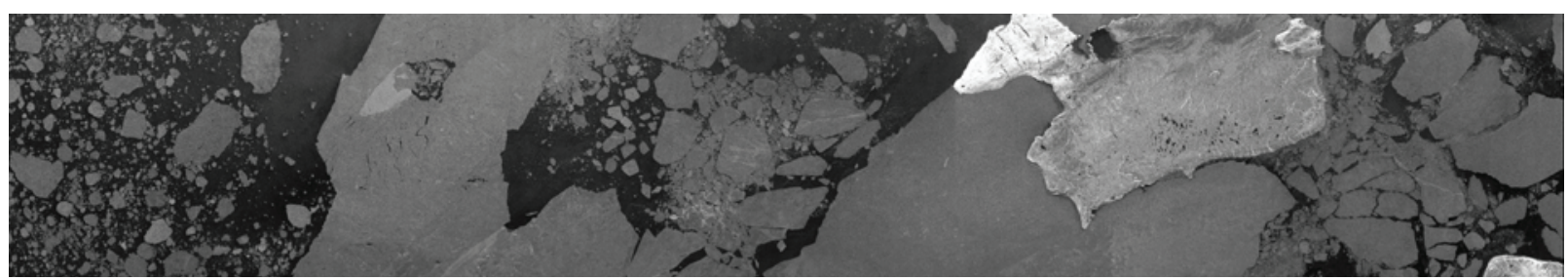

(a)

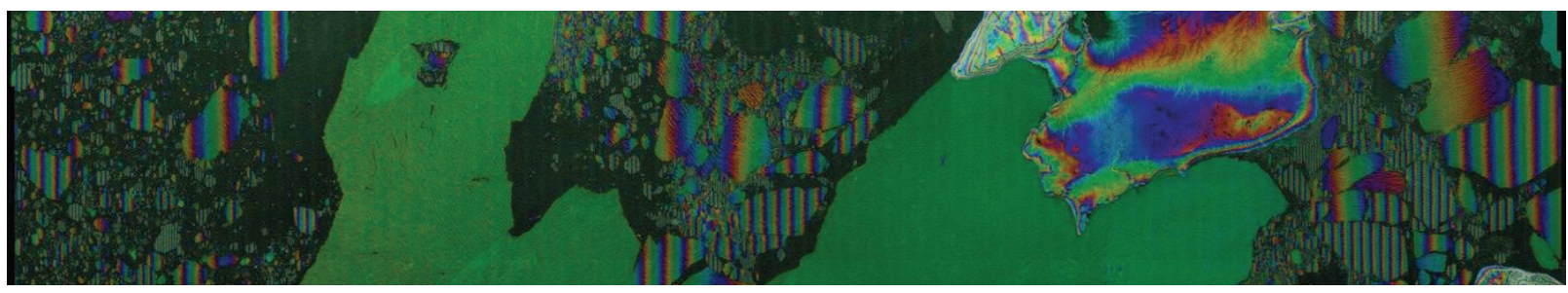

(b)

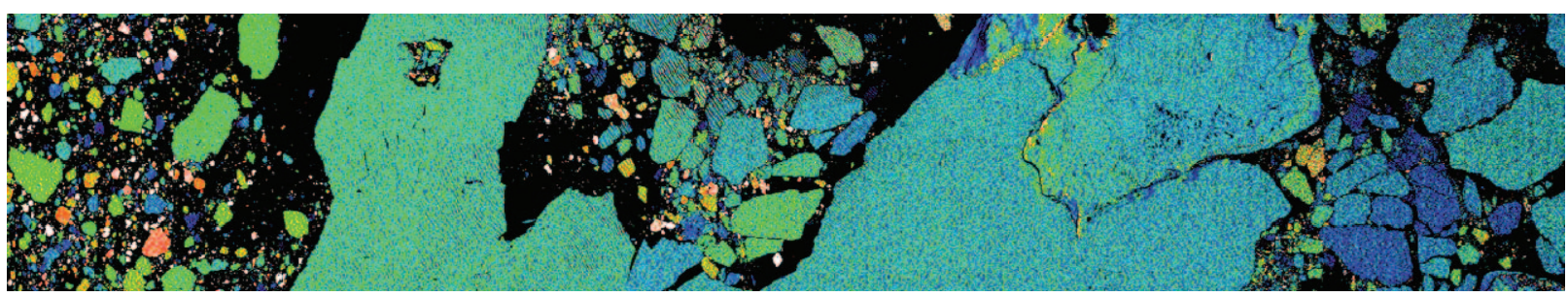

(c)

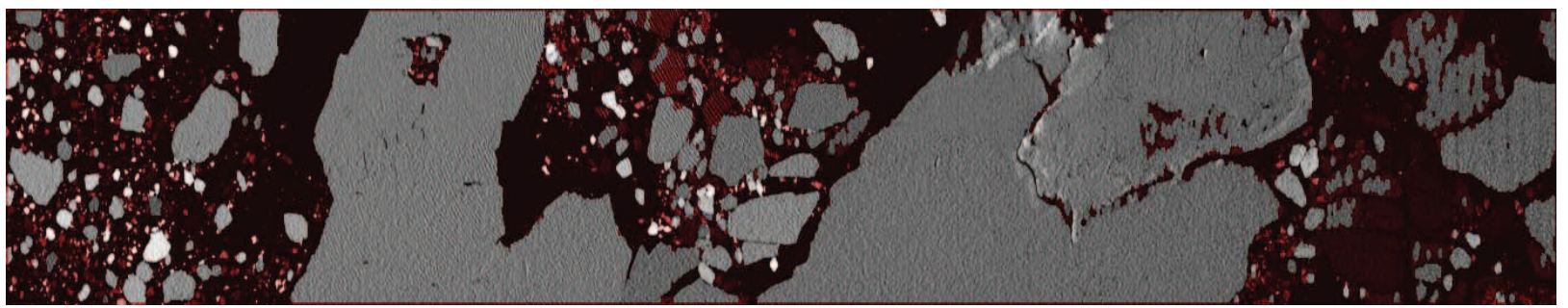

(d)

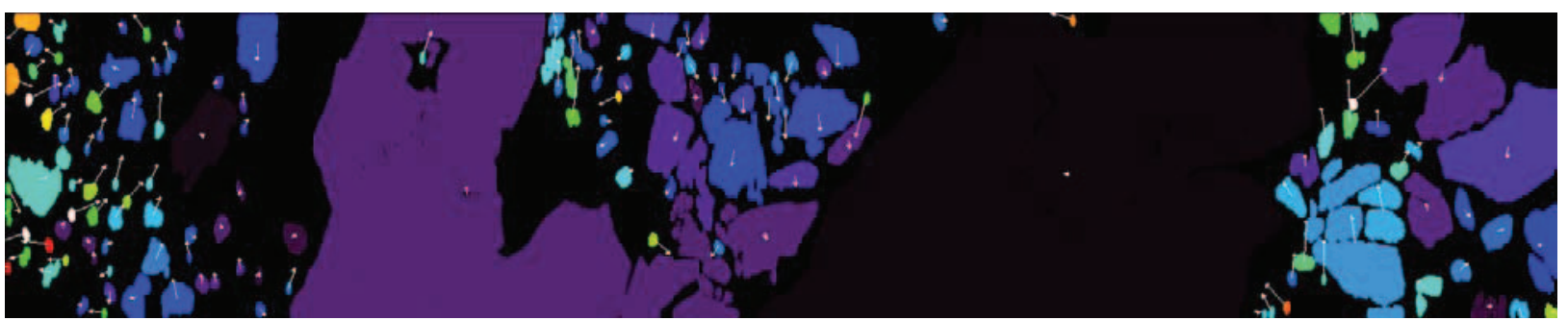

(e)

Fig. 5: Coastal area in North-East Greenland observed on $2^{\text {nd }}$ August, 2010 by TerraSAR-X and TanDEM-X. (a) third subswath radar brightness (complete ScanSAR scene includes 4 sub-swaths), (b) interferometric phase for the 2.6 second interval of the data acquisition, (c) sea-ice rotation map scaled from -0.005 to $+0.005 \mathrm{deg}$, (d) valid regions after segmentation, (e) estimated 2D translations scaled from 0 (black) to $0.5 \mathrm{~m}$ (white).

[5] S.N. Madsen. Estimating the Doppler Centroid of SAR Data. IEEE Trans. Aerosp. Electron. Syst., Vol. AES-25, Nr. 2, S. 134140, March 1989.

[6] Dana Ballard and Chris Brown. Computer Vision. PrenticeHall, 1982.
[7] P. Prats, M. Rodriguez-Cassola, L. Marotti, M. Nannini, S. Wollstadt, D. Schulze, N. Tous-Ramon, M. Younis, G. Krieger, A. Reigber, "TAXI: A versatile processing chain for experimental TanDEM-X product evaluation," Proceedings of IGARSS 2010, Honolulu, Hawaii, USA.

[8] P. Prats, R. Scheiber, J. Mittermayer, A. Meta, A. Moreira, „Processing of sliding spotlight and TOPS SAR data using baseband azimuth scaling," IEEE Trans. on Geosci. and Remote Sens., vol. 48, no. 2, pp. 770-780, Feb. 2010. 